\title{
Dixon 结式的三类多余因子
}

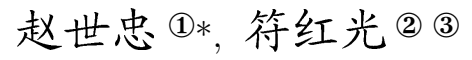

(1) 上海市高可信计算重点实验室, 华东师范大学, 上海 200062

(2) 电子科技大学计算机科学与工程学院, 成都 610054

(3) 中国科学院成都计算机应用研究所，成都 610041

*E-mail:szzhao@sei.ecnu.edu.cn

收稿日期: 2007-06-14; 接受日期: 2008-02-29

国家重点基础研究发展规划 (批准号: 2004CB318003)、中国科学院知识创新工程 (批准号: KJCX2-YW-S02)、国 家自然科学基金 (批准号: 90718041)、上海重点学科建设 (批准号: B412) 及华东师范大学博士启动基金 (批准号: 790013J4) 资助项目

摘要 Dixon 结式是一种基本消元方法, 它在自动控制、机器人等高技术领域中有着重 要应用。但是由于结式方法可能产生多余因子, 因此多余因子的产生机理一直是一大难题. 本文通过将 Dixon 结式表示成原多项式系统的线性组合, 发现了 Dixon 结式的部分多余因 子, 并且证明了其可由 Dixon 导出多项式的多余因子和 Dixon 矩阵的多余因子以及导出多 项式回代产生的多余因子三大部分组成.

关键词Dixon 结式 Dixon 矩阵 多余因子

$\mathrm{MSC}(2000)$ 主题分类 $00 \mathrm{~A} 06,13 \mathrm{~A} 50,13 \mathrm{P} 99,68 \mathrm{~W} 30$

\section{1 引言}

消去法理论的核心是通过消元求解非线性多项式方程组. 其主要的算法包括吴方法 ${ }^{[1]}$ 、 Gröbner 基方法 ${ }^{[2]}$ 和结式方法. 值得注意的是, 最近几年来, 国际上越来越多的学者把注意 力转到了结式方法. 事实上, 在几种结式方法中, Dixon 结式方法 ${ }^{[3-5]}$ 是最为有效和切实可 行的方法, 因为人们利用它的确解决了一些标高问题 ${ }^{[6-9]}$. 对于任意 $n$ 变元多项式系统

$$
f_{j}=\sum_{i_{1}=0}^{m_{1}} \ldots \sum_{i_{n}=0}^{m_{n}} a_{j, i_{1}, \ldots, i_{n}} x_{1}^{i_{1}} \ldots x_{n}^{i_{n}} \in \mathcal{C}[X], \quad 1 \leqslant j \leqslant n+1,
$$

其中 $X=\left\{x_{1}, \ldots, x_{n}\right\}$, 其结式为 0 是它有零点的一个充分必要条件. 在用 Dixon 结式方法 消元的过程中, 由于多余因子的存在, 不仅使得 Dixon 结式为 0 变成 (1) 式有零点的一个必 要但非充分的条件, 而且使得结式的计算变得困难, 导致有些问题无法求解. 针对这个问题,

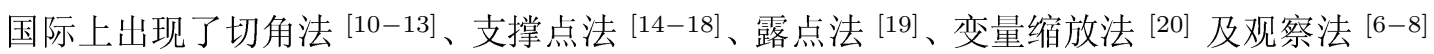
等, 遗憾的是它们只能处理某一特殊类型系统, 而杨路等人的 “聚筛法” [21] 是一种 “后验法”, 它通过 WR 相对分解去掉最后结果中的多余因子. 在下面部分, 本文将指出 Dixon 结式的 
多余因子最少由三大部分组成 (各部分的多余因子有可能为常数项): Dixon 导出多项式的多 余因子, Dixon 矩阵的多余因子以及最后导出多项式回代产生的多余因子, 并按照这 3 个部 分的顺序研究在构造过程中各部分多余因子的产生机理.

\section{$2 n$ 变元多项式系统的 Dixon 结式}

这一节回顾 $n$ 变元多项式系统 (1) 的 Dixon 结式 ${ }^{[3-5]}$.

设 $\bar{x}_{1}, \ldots, \bar{x}_{n}$ 是 $n$ 个新变元, 用 $f_{i}\left(\bar{x}_{1}, \ldots, \bar{x}_{k}, x_{k+1}, \ldots, x_{n}\right)$ 表示当 $x_{j}$ 取 $\bar{x}_{j}(1 \leqslant j \leqslant$ $k \leqslant n)$ 时 $f_{i}$ 的值, 则下列 $n+1$ 阶行列式:

$$
\Delta\left(x_{1}, \ldots, x_{n}, \bar{x}_{1}, \ldots, \bar{x}_{n}\right)=\left|\begin{array}{ccc}
f_{1}\left(x_{1}, x_{2}, \ldots, x_{n}\right) & \cdots & f_{n+1}\left(x_{1}, x_{2}, \ldots, x_{n}\right) \\
f_{1}\left(\bar{x}_{1}, x_{2}, \ldots, x_{n}\right) & \cdots & f_{n+1}\left(\bar{x}_{1}, x_{2}, \ldots, x_{n}\right) \\
f_{1}\left(\bar{x}_{1}, \bar{x}_{2}, \ldots, x_{n}\right) & \cdots & f_{n+1}\left(\bar{x}_{1}, \bar{x}_{2}, \ldots, x_{n}\right) \\
\vdots & & \vdots \\
f_{1}\left(\bar{x}_{1}, \bar{x}_{2}, \ldots, \bar{x}_{n}\right) & \cdots & f_{n+1}\left(\bar{x}_{1}, \bar{x}_{2}, \ldots, \bar{x}_{n}\right)
\end{array}\right|
$$

当 $x_{i}=\bar{x}_{i}(i=1, \ldots, n)$ 时, $\Delta\left(x_{1}, \ldots, x_{n}, \bar{x}_{1}, \ldots, \bar{x}_{n}\right)=0$, 所以 $\prod_{i=1}^{n}\left(\bar{x}_{i}-x_{i}\right)$ 是 $\Delta\left(x_{1}, \ldots\right.$, $\left.x_{n}, \bar{x}_{1}, \ldots, \bar{x}_{n}\right)$ 的因子. 令

$$
\delta\left(f_{1}, \ldots, f_{n+1}, x_{1}, \ldots, x_{n}, \bar{x}_{1}, \ldots, \bar{x}_{n}\right)=\frac{\Delta\left(x_{1}, \ldots, x_{n}, \bar{x}_{1}, \ldots, \bar{x}_{n}\right)}{\left(\bar{x}_{1}-x_{1}\right) \cdots\left(\bar{x}_{n}-x_{n}\right)},
$$

则 $\delta\left(f_{1}, \ldots, f_{n+1}, x_{1}, \ldots, x_{n}, \bar{x}_{1}, \ldots, \bar{x}_{n}\right)$ 是一个关于 $\left\{x_{1}, \ldots, x_{n}, \bar{x}_{1}, \ldots, \bar{x}_{n}\right\}$ 的多项式.

定义 2.1 多项式 $\delta\left(f_{1}, \ldots, f_{n+1}, x_{1}, \ldots, x_{n}, \bar{x}_{1}, \ldots, \bar{x}_{n}\right)$ 称为 $\left\{f_{1}, \ldots, f_{n+1}\right\}$ 关于 $\left\{x_{1}\right.$, $\left.\ldots, x_{n}, \bar{x}_{1}, \ldots, \bar{x}_{n}\right\}$ 的 Dixon 多项式.

若用 $\mathrm{X}$ 表示幕积

$$
x_{1}^{\sigma_{1}} x_{2}^{\sigma_{2}} \ldots x_{n}^{\sigma_{n}} \bar{x}_{1}^{u_{1}} \bar{x}_{2}^{u_{2}} \ldots \bar{x}_{n}^{u_{n}},
$$

则容易证明, $\delta$ 可以写成下列形式:

$$
\begin{aligned}
& \delta\left(f_{1}, \ldots, f_{n+1}, x_{1}, \ldots, x_{n}, \bar{x}_{1}, \ldots, \bar{x}_{n}\right) \\
& \quad=\sum_{u_{1}=0}^{n m_{1}-1} \sum_{u_{2}=0}^{(n-1) m_{2}-1} \cdots \sum_{u_{n}=0}^{m_{n}-1} \sum_{\sigma_{1}=0}^{m_{1}-1} \sum_{\sigma_{2}=0}^{m_{2}-1} \cdots \sum_{\sigma_{n}=0}^{n m_{n}-1} d_{\sigma_{1}, \sigma_{2}, \ldots, \sigma_{n}, u_{1}, u_{2}, \ldots, u_{n}} X,
\end{aligned}
$$

其中 $d_{\sigma_{1}, \sigma_{2}, \ldots, \sigma_{n}, u_{1}, u_{2}, \ldots, u_{n}}$ 是关于 (1) 式的系数的多项式.

将 $\delta$ 合并同类项, 按 $\left\{\bar{x}_{1}, \ldots, \bar{x}_{n}\right\}$ 的幕积展开, 则 $\delta$ 可表示成

$$
\begin{aligned}
& \delta\left(f_{1}, \ldots, f_{n+1}, x_{1}, \ldots, x_{n}, \bar{x}_{1}, \ldots, \bar{x}_{n}\right) \\
& \quad=\sum_{u_{1}=0}^{n m_{1}-1} \sum_{u_{2}=0}^{(n-1) m_{2}-1} \cdots \sum_{u_{n}=0}^{m_{n}-1} p_{u_{1}, u_{2}, \ldots, u_{n}} \bar{x}_{1}^{u_{1}} \bar{x}_{2}^{u_{2}} \cdots \bar{x}_{n}^{u_{n}},
\end{aligned}
$$

其中 $p_{u_{1}, u_{2}, \ldots, u_{n}}$ 是关于 $\left\{x_{1}, \ldots, x_{n}\right\}$ 的多项式.

定义 2.2 多项式 $p_{u_{1}, u_{2}, \ldots, u_{n}}$ 称为 $\left\{f_{1}, \ldots, f_{n+1}\right\}$ 关于 $\left\{x_{1}, \ldots, x_{n}\right\}$ 在 $\left\{u_{1}, \ldots, u_{n}\right\}$ 的 Dixon 导出多项式 ${ }^{[22]}$. 由导出多项式的全体构成的集合 $\left\{p_{u_{1}, u_{2}, \ldots, u_{n}} \mid 0 \leqslant u_{1} \leqslant n m_{1}-1,0 \leqslant\right.$ $\left.u_{2} \leqslant(n-1) m_{2}-1, \ldots, 0 \leqslant u_{n} \leqslant m_{n}-1\right\}$ 称为 $\left\{f_{1}, \ldots, f_{n+1}\right\}$ 关于 $\left\{x_{1}, \ldots, x_{n}\right\}$ 的 Dixon 导出多项式组 ${ }^{[21,22]}$. 
令

$$
\begin{gathered}
J=n ! \prod_{i=1}^{n} m_{i} \\
\boldsymbol{P}=\left[p_{0,0, \ldots, 0}, \ldots, p_{0,0, \ldots, m_{n}-1}, \ldots, p_{n m_{1}-1,(n-1) m_{2}-1, \ldots, m_{n}-1}\right]_{1 \times J} \\
\boldsymbol{X}=\left[\prod_{i=1}^{n} x_{i}^{i m_{i}-1}, \ldots, x_{1}^{m_{1}-1}, \ldots, x_{n}^{n m_{n}-1}, \ldots, 1\right]_{1 \times J}
\end{gathered}
$$

及

$$
\boldsymbol{Y}=\left[1, \ldots, \bar{x}_{n}^{m_{n}-1}, \ldots, \bar{x}_{1}^{n m_{1}-1}, \ldots, \prod_{i=1}^{n} \bar{x}_{i}^{(n-i+1) m_{i}-1}\right]_{1 \times J},
$$

则 $\delta$ 可以写成矩阵的形式

$$
\begin{aligned}
\delta & \left(f_{1}, \ldots, f_{n+1}, x_{1}, \ldots, x_{n}, \bar{x}_{1}, \ldots, \bar{x}_{n}\right) \\
& =\boldsymbol{Y} \cdot \boldsymbol{P}^{\mathrm{T}} \\
& =\boldsymbol{Y} \cdot \operatorname{Dixon}\left(f_{1}, \ldots, f_{n+1}\right) \cdot \boldsymbol{X}^{\mathrm{T}}
\end{aligned}
$$

其中系数矩阵为

$$
\operatorname{Dixon}\left(f_{1}, \ldots, f_{n+1}\right)
$$

$$
=\left(\begin{array}{ccc}
d_{m_{1}-1, \ldots, n m_{n}-1,0, \ldots, 0} & \ldots & d_{0, \ldots, 0,0, \ldots, 0} \\
\vdots & \ddots & \vdots \\
d_{m_{1}-1, \ldots, n m_{n}-1, n m_{1}-1, \ldots, m_{n}-1} & \ldots & d_{0, \ldots, 0, n m_{1}-1, \ldots, m_{n}-1}
\end{array}\right)_{J \times J} .
$$

定义 2.3 矩阵 $\operatorname{Dixon}\left(f_{1}, \ldots, f_{n+1}\right)$ 与它的的行列式分别称为 $\left\{f_{1}, \ldots, f_{n+1}\right\}$ 关于 $\left\{x_{1}\right.$, $\left.\ldots, x_{n}\right\}$ 的 Dixon 矩阵与 Dixon 结式.

例 2.1 考虑多项式组

$$
\left\{\begin{array}{l}
f(x, y)=-3 x y+1-2 z y \\
g(x, y)=3+2 z x \\
h(x, y)=(y-z)(-2+z) .
\end{array}\right.
$$

容易计算, 它的 Dixon 多项式为

$$
\delta(f, g, h, x, y, \bar{x}, \bar{y})=\frac{\left|\begin{array}{lll}
f(x, y) & g(x, y) & h(x, y) \\
f(\bar{x}, y) & g(\bar{x}, y) & h(\bar{x}, y) \\
f(\bar{x}, \bar{y}) & g(\bar{x}, \bar{y}) & h(\bar{x}, \bar{y})
\end{array}\right|}{(\bar{x}-x)(\bar{y}-y)}=p_{0,0}+p_{1,0} \bar{x},
$$

其中两个导出多项式分别为

$$
\left\{\begin{array}{l}
p_{0,0}=-4 z+2 z^{2}+8 z^{3}-4 z^{4}-18 y+9 z y, \\
p_{1,0}=-12 z y+6 z^{2} y-6 z^{3}+12 z^{2} .
\end{array}\right.
$$

由上述导出多项式组可构造下列 2 阶 Dixon 矩阵

$$
\operatorname{Dixon}(f, g, h)=\left(\begin{array}{cc}
-18+9 z & -4 z+2 z^{2}+8 z^{3}-4 z^{4} \\
-12 z+6 z^{2} & -6 z^{3}+12 z^{2}
\end{array}\right) .
$$


它的行列式, 即 Dixon 结式为

$$
|\operatorname{Dixon}(f, g, h)|=6 z^{2}\left(-11+4 z^{2}\right)(-2+z)^{2} .
$$

为方便后面的推导, 我们用 $f_{j, i}$ 表示 $f_{j}(1 \leqslant j \leqslant n+1)$ 关于 $x_{1}^{i}\left(0 \leqslant i \leqslant m_{1}\right)$ 的系数, 即

$$
f_{j, i}\left(x_{2}, \ldots, x_{n}\right)=\sum_{i_{2}=0}^{m_{2}} \ldots \sum_{i_{n}=0}^{m_{n}} a_{j, i, i_{2}, \ldots, i_{n}} x_{2}^{i_{2}} \ldots x_{n}^{i_{n}} .
$$

由 (3) 和 (4) 式, 可设

$$
\begin{aligned}
\delta\left(f_{1, i_{1}}, \ldots, f_{j-1, i_{j-1}}, f_{j+1, i_{j}}, \ldots, f_{n+1, i_{n}}, x_{2}, \ldots, x_{n}, \bar{x}_{2}, \ldots, \bar{x}_{n}\right) \\
\quad=\sum_{u_{2}=0}^{(n-1) m_{2}-1} \cdots \sum_{u_{n}=0}^{m_{n}-1} \sum_{\sigma_{2}=0}^{m_{2}-1} \cdots \sum_{\sigma_{n}=0}^{(n-1) m_{n}-1} d_{i_{1}, \ldots, i_{n}, \sigma_{2}, \ldots, \sigma_{n}, u_{2}, \ldots, u_{n}}^{j} x_{2}^{\sigma_{2}} \cdots x_{n}^{\sigma_{n}} \bar{x}_{2}^{u_{2}} \cdots \bar{x}_{n}^{u_{n}} \\
\quad=\sum_{u_{2}=0}^{(n-1) m_{2}-1} \cdots \sum_{u_{n}=0}^{m_{n}-1} p_{i_{1}, \ldots, i_{n}, u_{2}, \ldots, u_{n}}^{j} \bar{x}_{2}^{u_{2}} \cdots \bar{x}_{n}^{u_{n}},
\end{aligned}
$$

其中 $d_{i_{1}, \ldots, i_{n}, \sigma_{2}, \ldots, \sigma_{n}, u_{2}, \ldots, u_{n}}^{j}$ 是多项式组 $\left\{f_{1, i_{1}}, \ldots, f_{j-1, i_{j-1}}, f_{j+1, i_{j}}, \ldots, f_{n+1, i_{n}}\right\}$ 关于 $\left\{x_{2}, \ldots\right.$, $\left.x_{n}\right\}$ 的幂积的系数的多项式, $p_{i_{1}, \ldots, i_{n}, u_{2}, \ldots, u_{n}}^{j}$ 是 $\left\{f_{1, i_{1}}, \ldots, f_{j-1, i_{j-1}}, f_{j+1, i_{j}}, \ldots, f_{n+1, i_{n}}\right\}$ 关于 $\left\{x_{2}, \ldots, x_{n}\right\}$ 在 $\left\{u_{2}, \ldots, u_{n}\right\}$ 的 Dixon 导出多项式.

\section{3 导出多项式关于 $\left\{f_{1}, \ldots, f_{n+1}\right\}$ 的表示}

本节利用 $n-1$ 元多项式系统的 Dixon 导出多项式, 将 $n$ 元多项式系统 (1) 的 Dixon 导 出多项式表示成 $\left\{f_{1}, \ldots, f_{n+1}\right\}$ 的线性组合 ${ }^{[22]}$.

引理 3.1 设 $f(x, y)=\sum_{i_{1}=0}^{m} \sum_{i_{2}=0}^{m} \cdots \sum_{i_{n}=0}^{m} a_{i_{1}, i_{2}, \ldots, i_{n}}(y) x^{i_{1}+i_{2}+\ldots+i_{n}}$, 其中 $a_{i_{1}, i_{2}, \ldots, i_{n}}(y)$ 是关于 $y$ 的多项式, 且当 $x=y$ 时, $f(x, y) \equiv 0$, 则下列式子等价:

(i) $\sum_{i_{1}=0}^{m} \sum_{i_{2}=0}^{m} \cdots \sum_{i_{n}=0}^{m} a_{i_{1}, i_{2}, \ldots, i_{n}}(y) x^{i_{1}+i_{2}+\cdots+i_{n}} \frac{1}{x-y}$,

(ii) $\sum_{i_{1}=0}^{m} \sum_{i_{2}=0}^{m} \cdots \sum_{i_{n}=0}^{m} \sum_{u=0}^{i_{1}+i_{2}+\cdots+i_{n}-1} y^{i_{1}+i_{2}+\cdots+i_{n}-1-u} x^{u} a_{i_{1}, i_{2}, \ldots, i_{n}}(y)$,

(iii) $\sum_{i=0}^{n m} \sum_{u=0}^{i-1} y^{i-1-u} x^{u} \sum_{i_{1}+i_{2}+\cdots+i_{n}=i}^{0 \leqslant i_{1}, i_{2}, \ldots, i_{n} \leqslant m} a_{i_{1}, i_{2}, \ldots, i_{n}}(y)$.

引理 3.2 设 $a_{0}, a_{1}, \ldots, a_{m}$ 为多项式, 则有下列式子成立:

$$
\sum_{i=0}^{m} \sum_{u=0}^{i-1} x^{i-1-u} y^{u} a_{i}=\sum_{u=0}^{m-1} \sum_{i=u+1}^{m} x^{i-1-u} y^{u} a_{i} .
$$

引理 3.1 与 3.2 的证明过程见文献 [22].

定理 3.1 Dixon 导出多项式 $p_{u_{1}, u_{2}, \ldots, u_{n}}$ 可表示成 $\left\{f_{1}, \ldots, f_{n+1}\right\}$ 的线性组合

$$
\begin{aligned}
p_{u_{1}, u_{2}, \ldots, u_{n}}= & \sum_{i=u_{1}+1}^{n m_{1}} x_{1}^{i-1-u_{1}} \sum_{i_{1}+i_{2}+\ldots+i_{n}=i} p_{i_{1}, \ldots, i_{n}, u_{2}, \ldots, u_{n}}^{1} f_{1} \\
& +\cdots+(-1)^{n} \sum_{i=u_{1}+1}^{n m_{1}} x_{1}^{i-1-u_{1}} \sum_{i_{1}+i_{2}+\ldots+i_{n}=i} p_{i_{1}, \ldots, i_{n}, u_{2}, \ldots, u_{n}}^{n+1} f_{n+1},
\end{aligned}
$$

其中 $0 \leqslant u_{1} \leqslant n m_{1}-1,0 \leqslant u_{2} \leqslant(n-1) m_{2}-1, \ldots, 0 \leqslant u_{n} \leqslant m_{n}-1$.

证明 首先将 $(2)$ 式的 $\Delta\left(x_{1}, \ldots, x_{n}, \bar{x}_{1}, \ldots, \bar{x}_{n}\right)$ 按第 1 行展开, 即

$$
\delta\left(f_{1}, \ldots, f_{n+1}, x_{1}, \ldots, x_{n}, \bar{x}_{1}, \ldots, \bar{x}_{n}\right)
$$




$$
\begin{aligned}
& =\left(f_{1}\left(x_{1}, \ldots, x_{n}\right) \frac{\left|\begin{array}{ccc}
f_{2}\left(\bar{x}_{1}, x_{2}, \ldots, x_{n}\right) f_{3}\left(\bar{x}_{1}, x_{2}, \ldots, x_{n}\right) \cdots & f_{n+1}\left(\bar{x}_{1}, x_{2}, \ldots, x_{n}\right) \\
f_{2}\left(\bar{x}_{1}, \bar{x}_{2}, \ldots, x_{n}\right) f_{3}\left(\bar{x}_{1}, \bar{x}_{2}, \ldots, x_{n}\right) \cdots & f_{n+1}\left(\bar{x}_{1}, \bar{x}_{2}, \ldots, x_{n}\right) \\
\vdots & \vdots \\
f_{2}\left(\bar{x}_{1}, \bar{x}_{2}, \ldots, \bar{x}_{n}\right) f_{3}\left(\bar{x}_{1}, \bar{x}_{2}, \ldots, \bar{x}_{n}\right) \cdots f_{n+1}\left(\bar{x}_{1}, \bar{x}_{2}, \ldots, \bar{x}_{n}\right.
\end{array}\right|}{\left(\bar{x}_{2}-x_{2}\right) \cdots\left(\bar{x}_{n}-x_{n}\right)}\right. \\
& +\cdots+(-1)^{n} f_{n+1}\left(x_{1}, \ldots, x_{n}\right)
\end{aligned}
$$

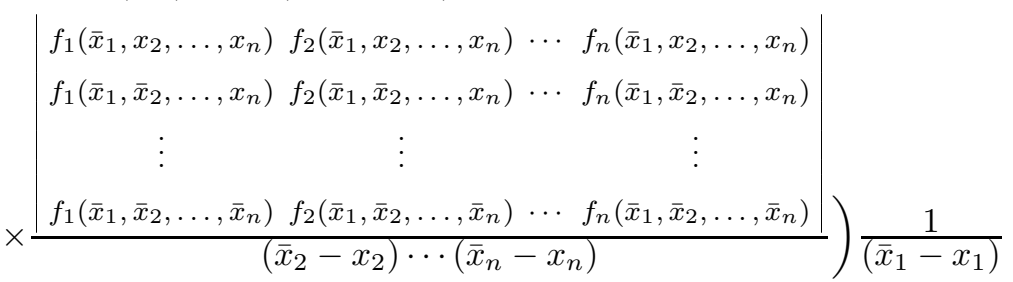

$$
\begin{aligned}
& =\left(f_{1}\left(x_{1}, \ldots, x_{n}\right)\right. \\
& \sum_{i_{1}=0}^{m_{1}} f_{2, i_{1}}\left(x_{2}, \ldots, x_{n}\right) \bar{x}_{1}^{i_{1}} \sum_{i_{2}=0}^{m_{1}} f_{3, i_{2}}\left(x_{2}, \ldots, x_{n}\right) \bar{x}_{1}^{i_{2}} \cdots \sum_{i_{n}=0}^{m_{1}} f_{n+1, i_{n}}\left(x_{2}, \ldots, x_{n}\right) \bar{x}_{1}^{i_{n}} \\
& \sum_{i_{1}=0}^{m_{1}} f_{2, i_{1}}\left(\bar{x}_{2}, \ldots, x_{n}\right) \bar{x}_{1}^{i_{1}} \sum_{i_{2}=0}^{m_{1}} f_{3, i_{2}}\left(\bar{x}_{2}, \ldots, x_{n}\right) \bar{x}_{1}^{i_{2}} \cdots \sum_{i_{n}=0}^{m_{1}} f_{n+1, i_{n}}\left(\bar{x}_{2}, \ldots, x_{n}\right) \bar{x}_{1}^{i_{n}} \\
& \times \frac{\left|\begin{array}{c}
\vdots \\
\sum_{i_{1}=0}^{m_{1}} f_{2, i_{1}}\left(\bar{x}_{2}, \ldots, \bar{x}_{n}\right) \bar{x}_{1}^{i_{1}} \sum_{i_{2}=0}^{m_{1}} f_{3, i_{2}}\left(\bar{x}_{2}, \ldots, \bar{x}_{n}\right) \bar{x}_{1}^{i_{2}} \cdots \sum_{i_{n}=0}^{m_{1}} f_{n+1, i_{n}}\left(\bar{x}_{2}, \ldots, \bar{x}_{n}\right) \bar{x}_{1}^{i_{n}}
\end{array}\right|}{\left(\bar{x}_{2}-x_{2}\right) \cdots\left(\bar{x}_{n}-x_{n}\right)} \\
& +\cdots+(-1)^{n} f_{n+1}\left(x_{1}, \ldots, x_{n}\right)
\end{aligned}
$$

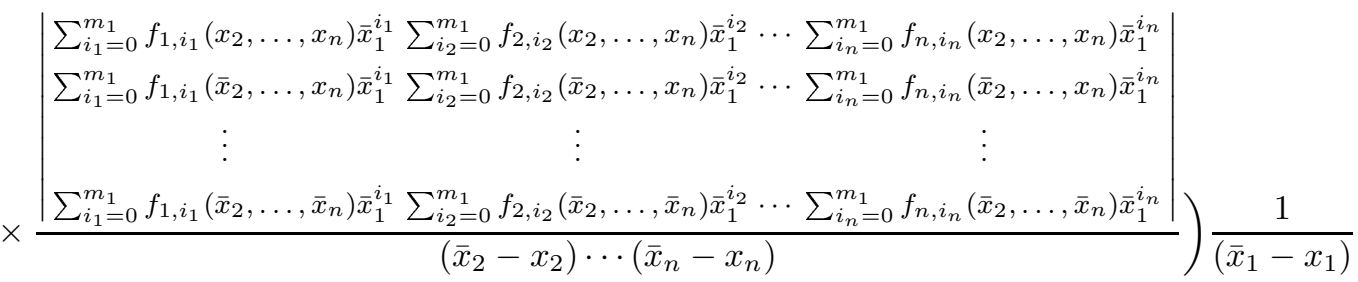

$$
\begin{aligned}
& =\sum_{i_{1}=0}^{m_{1}} \sum_{i_{2}=0}^{m_{1}} \cdots \sum_{i_{n}=0}^{m_{1}} \bar{x}_{1}^{i_{1}+i_{2}+\cdots+i_{n}}\left(f_{1}\left(x_{1}, \ldots, x_{n}\right) \delta\left(f_{2, i_{1}}, \ldots, f_{n+1, i_{n}}, x_{2}, \ldots, x_{n}, \bar{x}_{2}, \cdots, \bar{x}_{n}\right)\right. \\
& \left.+\cdots+(-1)^{n} f_{n+1}\left(x_{1}, \ldots, x_{n}\right) \delta\left(f_{1, i_{1}}, \ldots, f_{n, i_{n}}, x_{2}, \ldots, x_{n}, \bar{x}_{2}, \ldots, \bar{x}_{n}\right)\right) \frac{1}{\left(\bar{x}_{1}-x_{1}\right)} .
\end{aligned}
$$

其次由引理 $3.1,(19)$ 式的 $\frac{1}{\left(\bar{x}_{1}-x_{1}\right)}$ 可以消去, 从而化为

$$
\begin{aligned}
\delta\left(f_{1}, \ldots, f_{n+1}, x_{1}, \ldots, x_{n}, \bar{x}_{1}, \ldots, \bar{x}_{n}\right) & \sum_{i_{1}=0}^{m_{1}} \sum_{i_{2}=0}^{m_{1}} \cdots \sum_{i_{n}=0}^{m_{1}} \sum_{u_{1}=0}^{i_{1}+i_{2}+\cdots+i_{n}-1} x_{1}^{i_{1}+i_{2}+\cdots+i_{n}-1-u_{1}} \bar{x}_{1}^{u_{1}}\left(f _ { 1 } ( x _ { 1 } , \ldots , x _ { n } ) \delta \left(f_{2, i_{1}}, \ldots,\right.\right. \\
& \left.f_{n+1, i_{n}}, x_{2}, \ldots, x_{n}, \bar{x}_{2}, \ldots, \bar{x}_{n}\right)+\cdots+(-1)^{n} f_{n+1}\left(x_{1}, \ldots, x_{n}\right) \delta\left(f_{1, i_{1}}, \ldots, f_{n, i_{n}}\right. \\
& \left.\left.x_{2}, \ldots, x_{n}, \bar{x}_{2}, \ldots, \bar{x}_{n}\right)\right) \\
= & \sum_{i=0}^{n m_{1}} \sum_{u_{1}=0}^{i-1} x_{1}^{i-1-u_{1}} \bar{x}_{1}^{u_{1}}\left(f _ { 1 } ( x _ { 1 } , \ldots , x _ { n } ) \sum _ { i _ { 1 } + i _ { 2 } + \ldots + i _ { n } = i } \delta \left(f_{2, i_{1}}, \ldots, f_{n+1, i_{n}}, x_{2}, \ldots, x_{n},\right.\right. \\
& \left.\bar{x}_{2}, \ldots, \bar{x}_{n}\right)+\cdots+(-1)^{n} f_{n+1}\left(x_{1}, \ldots, x_{n}\right) \sum_{i_{1}+i_{2}+\cdots+i_{n}=i} \delta\left(f_{1, i_{1}}, \ldots, f_{n, i_{n}}, x_{2},\right.
\end{aligned}
$$




$$
\begin{aligned}
& \left.\left.\ldots, x_{n}, \bar{x}_{2}, \ldots, \bar{x}_{n}\right)\right) \\
= & \sum_{i=0 u_{1}=0}^{n m_{1}} \sum_{1}^{i-1} x_{1}^{i-1-u_{1}} \bar{x}_{1}^{u_{1}} \sum_{i_{1}+i_{2}+\cdots+i_{n}=i} \sum_{u_{2}=0}^{(n-1) m_{2}-1} \cdots \sum_{u_{n}=0}^{m_{n}-1} \sum_{\sigma_{2}=0}^{m_{2}-1} \ldots \sum_{\sigma_{n}=0}^{(n-1) m_{n}-1}\left(d_{i_{1}, \ldots, i_{n}, \sigma_{2}, \ldots, \sigma_{n}, u_{2}, \ldots, u_{n}}^{1} \cdots x_{n}^{\sigma_{n}} f_{1} \bar{x}_{2}^{u_{2}} \ldots \bar{x}_{n}^{u_{n}}+\cdots+(-1)^{n} d_{i_{1}, \ldots, i_{n}, \sigma_{2}, \ldots, \sigma_{n}, u_{2}, \cdots, u_{n}}^{n+1} x_{2}^{\sigma_{2}} \ldots x_{n}^{\sigma_{n}} f_{n+1} \bar{x}_{2}^{u_{2}} \ldots \bar{x}_{n}^{u_{n}}\right),(21)
\end{aligned}
$$

然后由引理 $3.2, \delta$ 可写成

$$
\begin{aligned}
& \delta\left(f_{1}, \ldots, f_{n+1}, x_{1}, \ldots, x_{n}, \bar{x}_{1}, \ldots, \bar{x}_{n}\right) \\
& =\sum_{u_{1}=0}^{n m_{1}-1} \sum_{i=u_{1}+1}^{n m_{1}} x_{1}^{i-1-u_{1}} \bar{x}_{1}^{u_{1}} \sum_{i_{1}+i_{2}+\cdots+i_{n}=i} \sum_{u_{2}=0}^{(n-1) m_{2}-1} \cdots \sum_{u_{n}=0}^{m_{n}-1} \sum_{\sigma_{2}=0}^{m_{2}-1} \cdots \sum_{\sigma_{n}=0}^{(n-1) m_{n}-1} \\
& \left(d_{i_{1}, \ldots, i_{n}, \sigma_{2}, \ldots, \sigma_{n}, u_{2}, \ldots, u_{n}}^{1} x_{2}^{\sigma_{2}} \cdots x_{n}^{\sigma_{n}} f_{1} \bar{x}_{2}^{u_{2}} \cdots \bar{x}_{n}^{u_{n}}+\cdots\right. \\
& \left.+(-1)^{n} d_{i_{1}, \ldots, i_{n}, \sigma_{2}, \ldots, \sigma_{n}, u_{2}, \ldots, u_{n}}^{n+1} x_{2}^{\sigma_{2}} \cdots x_{n}^{\sigma_{n}} f_{n+1} \bar{x}_{2}^{u_{2}} \cdots \bar{x}_{n}^{u_{n}}\right) \\
& =\sum_{u_{1}=0}^{n m_{1}-1} \sum_{u_{2}=0}^{(n-1) m_{2}-1} \cdots \sum_{u_{n}=0}^{m_{n}-1}\left(\sum_{i=u_{1}+1}^{n m_{1}} x_{1}^{i-1-u_{1}} f_{1} \sum_{i_{1}+i_{2}+\cdots+i_{n}=i} \sum_{\sigma_{2}=0}^{m_{2}-1} \cdots \sum_{\sigma_{n}=0}^{(n-1) m_{n}-1}\right. \\
& d_{i_{1}, \ldots, i_{n}, \sigma_{2}, \ldots, \sigma_{n}, u_{2}, \ldots, u_{n}}^{1} x_{2}^{\sigma_{2}} \ldots x_{n}^{\sigma_{n}}+\cdots+(-1)^{n} \sum_{i=u_{1}+1}^{n m_{1}} x_{1}^{i-1-u_{1}} f_{n+1} \sum_{i_{1}+i_{2}+\cdots+i_{n}=i} \\
& \left.\sum_{\sigma_{2}=0}^{m_{2}-1} \cdots \sum_{\sigma_{n}=0}^{(n-1) m_{n}-1} d_{i_{1}, \ldots, i_{n}, \sigma_{2}, \ldots, \sigma_{n}, u_{2}, \cdots, u_{n}}^{n+1} x_{2}^{\sigma_{2}} \cdots x_{n}^{\sigma_{n}}\right) \bar{x}_{1}^{u_{1}} \bar{x}_{2}^{u_{2}} \ldots \bar{x}_{n}^{u_{n}} .
\end{aligned}
$$

再由 (4) 式与上式可推得

$$
\begin{aligned}
p_{u_{1}, u_{2}, \ldots, u_{n}} & \sum_{i=u_{1}+1}^{n m_{1}} x_{1}^{i-1-u_{1}} \sum_{i_{1}+i_{2}+\ldots+i_{n}=i} \sum_{\sigma_{2}=0}^{m_{2}-1} \ldots \sum_{\sigma_{n}=0}^{(n-1) m_{n}-1} d_{i_{1}, \ldots, i_{n}, \sigma_{2}, \ldots, \sigma_{n}, u_{2}, \ldots, u_{n}}^{1} x_{2}^{\sigma_{2}} \ldots x_{n}^{\sigma_{n}} f_{1} \\
& +\cdots+(-1)^{n} \sum_{i=u_{1}+1}^{n m_{1}} x_{1}^{i-1-u_{1}} \sum_{i_{1}+i_{2}+\ldots+i_{n}=i} \sum_{\sigma_{2}=0}^{m_{2}-1} \ldots \sum_{\sigma_{n}=0}^{(n-1) m_{n}-1} d_{i_{1}, \ldots, i_{n}, \sigma_{2}, \ldots, \sigma_{n}, u_{2}, \ldots, u_{n}}^{n+1} \\
& \quad \cdots x_{n}^{\sigma_{2}} \cdots x_{n+1}^{\sigma_{n}} .
\end{aligned}
$$

最后通过 (15) 与 (16) 式, 可将上式化成 (18) 式.

设 Dixon 导出多项式 $p_{u_{1}, u_{2}, \ldots, u_{n}}=\sum_{j=1}^{n+1}(-1)^{j+1} k_{j, u_{1}, u_{2}, \ldots, u_{n}} f_{j}$, 则由上述定理我们可以 推出以下计算 $k_{j, u_{1}, u_{2}, \ldots, u_{n}}$ 的算法:

\section{算法 3.1}

第 1 步. 初始化

第 2 步. 循环迭代

$$
k_{j, u_{1}, u_{2}, \ldots, u_{n}}=\sum_{i_{1}+i_{2}+\cdots+i_{n}=u_{1}+1} p_{i_{1}, i_{2}, \ldots, i_{n}, u_{2}, \ldots, u_{n}}^{j} .
$$

$$
\begin{aligned}
\text { for } & u_{1} \text { from } n m_{1}-2 \text { to } 0 \text { by }-1 \\
& k_{j, u_{1}, u_{2}, \ldots, u_{n}}=k_{j, u_{1}, u_{2}, \ldots, u_{n}}+x_{1} k_{j, u_{1}+1, u_{2}, \ldots, u_{n}} .
\end{aligned}
$$

例 3.1 对于例 2.1 中的导出多项式组 (13), 我们可以将其表示成 $\{f, g, h\}$ 的线性组合

$$
\left\{\begin{array}{l}
p_{0,0}=\left(-4 z+2 z^{2}\right) f+\left(-6 z+3 z^{2}\right) g+\left(4 z^{2}+9+6 z x\right) h, \\
p_{1,0}=0 f+0 g+6 z h .
\end{array}\right.
$$




\section{Dixon 结式的多余因子}

这一节, 我们利用前面的知识导出 Dixon 结式的 3 部分多余因子.

\subsection{Dixon 导出多项式的多余因子}

为方便下面的推导, 我们将导出多项式列表 (6) 写成

$$
\overrightarrow{\boldsymbol{P}}=\left[P_{1}, P_{2}, \ldots, P_{J}\right] .
$$

由定理 3.1 知, 存在 $k_{i, j}(\in \mathcal{C}[X])$, 使得

$$
P_{i}=k_{i, 1} f_{1}+k_{i, 2} f_{2}+\cdots+k_{i, n+1} f_{n+1},
$$

其中 $1 \leqslant i \leqslant J, 1 \leqslant j \leqslant n+1$.

对于任意 $l$ 个多项式 $u_{1}, u_{2}, \ldots, u_{l} \in \mathcal{C}[X]$, 用 $\operatorname{GCD}\left(u_{1}, u_{2}, \ldots, u_{l}\right)$ 表示它们的不包含任 一变量 $x_{i}(1 \leqslant i \leqslant n)$ 的最大公因子, 则由 $(24)$ 式可得

定理 4.1 Dixon 导出多项式 $P_{i}(1 \leqslant i \leqslant J)$ 可产生多余因子

$$
e_{i}=\operatorname{GCD}\left(k_{i, 1}, k_{i, 2}, \ldots, k_{i, n+1}\right) \text {. }
$$

由于有 $J$ 个导出多项式, 所以有

定理 4.2 Dixon 导出多项式组 $\left\{P_{1}, P_{2}, \ldots, P_{J}\right\}$ 可产生多余因子

$$
E_{1}=\prod_{i=1}^{J} e_{i}=\prod_{i=1}^{J} \operatorname{GCD}\left(k_{i, 1}, k_{i, 2}, \ldots, k_{i, n+1}\right) .
$$

例 4.1 对于例 3.1 中的两个导出多项式 (13)', 可分别用 $P_{1}$ 和 $P_{2}$ 表示它们. 它们的关 于 $\{f, g, h\}$ 的系数的最大公因子 $\mathrm{GCD}$ 分别为

$$
\left\{\begin{array}{l}
e_{1}=1 \\
e_{2}=6 z
\end{array}\right.
$$

因此两个导出多项式可产生多余因子

$$
E_{1}=\prod_{i=1}^{2} e_{i}=6 z .
$$

将 $E_{1}$ 去掉, Dixon 结式 $|\operatorname{Dixon}(f, g, h)|$ 变为

$$
D_{1}=\frac{|\operatorname{Dixon}(f, g, h)|}{E_{1}}=z\left(-11+4 z^{2}\right)(-2+z)^{2} .
$$

\subsection{Dixon 矩阵的多余因子}

为方便讨论, 用 $d_{i, j}$ 表示矩阵 (11) 中的第 $i$ 行第 $j$ 列元素. 即

$$
\operatorname{Dixon}\left(f_{1}, \ldots, f_{n+1}\right)=\left(\begin{array}{ccc}
d_{1,1} & \cdots & d_{1, J} \\
\vdots & \ddots & \vdots \\
d_{J, 1} & \cdots & d_{J, J}
\end{array}\right)_{J \times J} .
$$

首先去掉导出多项式的多余因子. 令

$$
P_{i}^{\prime}=\frac{P_{i}}{e_{i}},
$$

则可将

$$
\left[P_{1}^{\prime}, P_{2}^{\prime}, \ldots, P_{J}^{\prime}\right]
$$


写成矩阵形式

$$
\left[P_{1}^{\prime}, P_{2}^{\prime}, \ldots, P_{J}^{\prime}\right]^{\mathrm{T}}=\operatorname{Dixon}^{\prime}\left(f_{1}, \ldots, f_{n+1}\right) \cdot \boldsymbol{X}^{\mathrm{T}}
$$

其中

$$
\operatorname{Dixon}^{\prime}\left(f_{1}, \ldots, f_{n+1}\right)=\begin{gathered}
\prod_{i=1}^{n} x_{i}^{i m_{i}-1} \\
P_{1}^{\prime} \\
P_{2}^{\prime} \\
\vdots \\
d_{j}^{\prime}
\end{gathered}\left(\begin{array}{ccc}
d_{1,1}^{\prime} & \cdots & 1 \\
d_{2,1}^{\prime} & \cdots & d_{1, J}^{\prime} \\
\vdots & & \vdots \\
d_{J, 1}^{\prime} & \cdots & d_{J, J}^{\prime}
\end{array}\right) .
$$

多项式列表 (31) 与单项式列表 (7) 分别是它的行指标与列指标, 并且 $d_{i, j}^{\prime}=\frac{d_{i, j}}{e_{i}}, 1 \leqslant$ $i, j \leqslant J$.

这时有

$$
\left|\operatorname{Dixon}^{\prime}\left(f_{1}, \ldots, f_{n+1}\right)\right| \cdot E_{1}=\left|\operatorname{Dixon}\left(f_{1}, \ldots, f_{n+1}\right)\right| .
$$

对于任一矩阵 $M$, 我们用 $M_{i, j}$ 来表示其第 $i$ 行第 $j$ 列的代数余子式. 同时用 $D^{\prime}$ 表示 矩阵 $\operatorname{Dixon}^{\prime}\left(f_{1}, \ldots, f_{n+1}\right)$, 则有

定理 4.3 行列式 $\left|D^{\prime}\right|$ 可表示成多项式组 (31) 的线性组合

$$
\left|D^{\prime}\right|=D_{1, J}^{\prime} P_{1}^{\prime}+D_{2, J}^{\prime} P_{2}^{\prime}+\cdots+D_{J, J}^{\prime} P_{J}^{\prime} .
$$

证明 首先由行列式的性质有

$$
\left\{\begin{array}{c}
\sum_{i=1}^{J} d_{i, 1}^{\prime} D_{i, J}^{\prime}=0, \\
\ldots \\
\sum_{i=1}^{J} d_{i, J-1}^{\prime} D_{i, J}^{\prime}=0, \\
\sum_{i=1}^{J} d_{i, J}^{\prime} D_{i, J}^{\prime}=\left|D^{\prime}\right| .
\end{array}\right.
$$

然后将上式的第 $j$ 个等式两边同乘以矩阵 $D^{\prime}$ 的第 $j$ 列的列指标, 其中 $1 \leqslant j \leqslant J$, 则有

$$
\left\{\begin{array}{c}
\prod_{i=1}^{n} x_{i}^{i m_{i}-1} \sum_{i=1}^{J} d_{i, 1}^{\prime} D_{i, J}^{\prime}=0, \\
\cdots \\
x_{n} \sum_{i=1}^{J} d_{i, J-1}^{\prime} D_{i, J}^{\prime}=0 \\
\sum_{i=1}^{J} d_{i, J}^{\prime} D_{i, J}^{\prime}=\left|D^{\prime}\right| .
\end{array}\right.
$$

再将 $J$ 个等式的左右两边分别相加, 可得

$$
\prod_{i=1}^{n} x_{i}^{i m_{i}-1} \sum_{i=1}^{J} d_{i, 1}^{\prime} D_{i, J}^{\prime}+\cdots+x_{n} \sum_{i=1}^{J} d_{i, J-1}^{\prime} D_{i, J}^{\prime}+\sum_{i=1}^{J} d_{i, J}^{\prime} D_{i, J}^{\prime}=\left|D^{\prime}\right| .
$$


最后将上式左边重新合并化简, 就可化为 (35) 式右边的式子.

由上面的定理可推得 Dixon 结式 $\left|\operatorname{Dixon}\left(f_{1}, \ldots, f_{n+1}\right)\right|$ 的第 2 部分多余因子.

定理 $4.4\left|D^{\prime}\right|$ 的 $J$ 个代数余子式的最大公因子

$$
E_{2}=\operatorname{GCD}\left(D_{1, J}^{\prime}, D_{2, J}^{\prime}, \ldots, D_{J, J}^{\prime}\right)
$$

为 Dixon 结式 $\left|\operatorname{Dixon}\left(f_{1}, \ldots, f_{n+1}\right)\right|$ 的多余因子.

例 4.2 若对例 3.1 中的两个导出多项式 (13) 分别去掉 (27) 式中的多余因子, 则它们 成为

$$
\left\{\begin{array}{l}
P_{1}^{\prime}=\frac{P_{1}}{e_{1}}=\left(-4 z+2 z^{2}\right) f+\left(-6 z+3 z^{2}\right) g+\left(4 z^{2}+9+6 z x\right) h, \\
P_{2}^{\prime}=\frac{P_{2}}{e_{2}}=0 f+0 g+h,
\end{array}\right.
$$

即

$$
\left\{\begin{array}{l}
P_{1}^{\prime}=-4 z+2 z^{2}+8 z^{3}-4 z^{4}-18 y+9 z y \\
P_{2}^{\prime}=-2 y+z y+2 z-z^{2}
\end{array}\right.
$$

由上述多项式组的 $x$ 和 $y$ 的幕积的系数可构造下列矩阵:

$$
\operatorname{Dixon}^{\prime}(f, g, h)=D^{\prime}=\left(\begin{array}{cc}
-18+9 z & -4 z+2 z^{2}+8 z^{3}-4 z^{4} \\
-2+z & -z^{2}+2 z
\end{array}\right),
$$

这时矩阵的行列式 $\left|\operatorname{Dixon}^{\prime}(f, g, h)\right|$ 可由 $\left\{P_{1}^{\prime}, P_{2}^{\prime}\right\}$ 及矩阵的关于最后一列的两个代数余子式 $\left\{D_{1,4}^{\prime}, D_{2,4}^{\prime}\right\}$ 表示, 即

$$
\begin{aligned}
\left|\operatorname{Dixon}^{\prime}(f, g, h)\right| & =D_{1,2}^{\prime} P_{1}^{\prime}+D_{2,2}^{\prime} P_{2}^{\prime} \\
& =z\left(-11+4 z^{2}\right)(-2+z)^{2},
\end{aligned}
$$

其中

$$
\left\{\begin{array}{l}
D_{1,2}^{\prime}=-(-2+z) \\
D_{2,2}^{\prime}=-18+9 z
\end{array}\right.
$$

所以

$$
E_{2}=\operatorname{GCD}\left(D_{1,2}^{\prime}, D_{2,2}^{\prime}\right)=-2+z
$$

为 $\left|\operatorname{Dixon}^{\prime}(f, g, h)\right|$ 的多余因子. 去掉 $E_{2}$ 后, $\left|\operatorname{Dixon}^{\prime}(f, g, h)\right|$ 就变为

$$
D_{2}=\frac{\left|\operatorname{Dixon}^{\prime}(f, g, h)\right|}{E_{2}}=z\left(-11+4 z^{2}\right)(-2+z) .
$$

\section{3 导出多项式回代后的多余因子}

这一节, 讨论 Dixon 结式的第 3 部分多余因子.

由等式 $(35),(30)$ 及 $(24)$, 有 


$$
\begin{aligned}
\frac{\left|\operatorname{Dixon}^{\prime}\left(f_{1}, \ldots, f_{n+1}\right)\right|}{E_{2}}= & \frac{D_{1, J}^{\prime} P_{1}^{\prime}+D_{2, J}^{\prime} P_{2}^{\prime}+\ldots+D_{J, J}^{\prime} P_{J}^{\prime}}{E_{2}} \\
= & \frac{D_{1, J}^{\prime}}{E_{2}} \frac{P_{1}}{e_{1}}+\frac{D_{2, J}^{\prime}}{E_{2}} \frac{P_{2}}{e_{2}}+\cdots+\frac{D_{J, J}^{\prime}}{E_{2}} \frac{P_{J}}{e_{J}} \\
= & \frac{D_{1, J}^{\prime}}{E_{2}} \frac{\left(k_{1,1} f_{1}+k_{1,2} f_{2}+\cdots+k_{1, n+1} f_{n+1}\right)}{e_{1}} \\
& +\frac{D_{2, J}^{\prime}}{E_{2}} \frac{\left(k_{2,1} f_{1}+k_{2,2} f_{2}+\cdots+k_{2, n+1} f_{n+1}\right)}{e_{2}} \\
& +\cdots+\frac{D_{J, J}^{\prime}}{E_{2}} \frac{\left(k_{J, 1} f_{1}+k_{J, 2} f_{2}+\cdots+k_{J, n+1} f_{n+1}\right)}{e_{J}} \\
= & K_{1} f_{1}+K_{2} f_{2}+\cdots+K_{n+1} f_{n+1},
\end{aligned}
$$

其中

$$
K_{i}=\frac{D_{1, J}^{\prime}}{E_{2}} \frac{k_{1, i}}{e_{1}}+\frac{D_{2, J}^{\prime}}{E_{2}} \frac{k_{2, i}}{e_{2}}+\cdots+\frac{D_{J, J}^{\prime}}{E_{2}} \frac{k_{J, i}}{e_{J}}, \quad 1 \leqslant i \leqslant n+1 .
$$

于是, 由 (41) 式可得 Dixon 结式 $\left|\operatorname{Dixon}\left(f_{1}, \ldots, f_{n+1}\right)\right|$ 的第 3 部分多余因子.

定理 4.5

$$
E_{3}=\operatorname{GCD}\left(K_{1}, K_{2}, \ldots, K_{n+1}\right)
$$

为 (41) 式的多余因子.

例 4.3 对于 (40) 式, 可由 (37)-(39) 式计算得出

$$
D_{2}=\frac{\left|D^{\prime}\right|}{E_{2}}=K_{1} f+K_{2} g+K_{3} h=z\left(-11+4 z^{2}\right)(-2+z),
$$

其中

$$
\left\{\begin{array}{l}
K_{1}=-(-4+2 z) z \\
K_{2}=-(-6+3 z) z \\
K_{3}=-(4 z+6 x) z
\end{array}\right.
$$

所以

$$
E_{3}=\operatorname{GCD}\left(K_{1}, K_{2}, K_{3}\right)=z
$$

为多余因子. 去掉 $E_{3}$ 后, $D_{2}$ 就变为

$$
D_{3}=\frac{D_{2}}{E_{3}}=(-2+z)\left(-11+4 z^{2}\right) .
$$

事实上, $D_{3}$ 是 $\{f, g, h\}$ 关于 $\{x, y\}$ 的结式. 它为 0 是 $\{f, g, h\}$ 有公共零点的一个充分 必要条件.

事实上, 由等式 (34) 与 (41) 可得出

$$
\begin{aligned}
\left|\operatorname{Dixon}\left(f_{1}, \ldots, f_{n+1}\right)\right| & =\left|\operatorname{Dixon}^{\prime}\left(f_{1}, \ldots, f_{n+1}\right)\right| E_{1} \\
& =E_{1} \cdot E_{2} \cdot E_{3} \cdot\left(\frac{K_{1}}{E_{3}} f_{1}+\frac{K_{2}}{E_{3}} f_{2}+\cdots+\frac{K_{n+1}}{E_{3}} f_{n+1}\right),
\end{aligned}
$$

其中

$$
\operatorname{GCD}\left(\frac{K_{1}}{E_{3}}, \frac{K_{2}}{E_{3}}, \ldots, \frac{K_{n+1}}{E_{3}}\right)=1
$$


于是, 由上式可得下列本文的两个主要结论:

\section{定理 4.6}

$$
E_{1} \cdot E_{2} \cdot E_{3}
$$

为 $\operatorname{Dixon}$ 结式 $\left|\operatorname{Dixon}\left(f_{1}, \ldots, f_{n+1}\right)\right|$ 的三类多余因子.

定理 4.7 若理想 $\left\langle f_{1}, \ldots, f_{n+1}\right\rangle$ 中的每个多项式关于 $\left\{f_{1}, \ldots, f_{n+1}\right\}$ 的表示形式是唯 一的, 则 Dixon 结式 $\left|\operatorname{Dixon}\left(f_{1}, \ldots, f_{n+1}\right)\right|$ 的三类多余因子

$$
E_{1} \cdot E_{2} \cdot E_{3}
$$

为其全部多余因子.

\section{5 算法}

本节讨论去掉三类多余因子的算法.

首先设导出多项式组如 $(6)^{\prime}$ 式所示, 并且其为 Dixon 矩阵 $D$ 的行指标, 而 1 为 Dixon 矩阵 $D$ 的最后一列的列指标, 则计算无三类多余因子的 Dixon 结式的算法如下:

\section{算法 5.1}

$$
\begin{aligned}
& \text { for } i \text { from } 1 \text { to } J \\
& \boldsymbol{k}_{i}=\left(k_{i, 1}, \ldots, k_{i, n+1}\right) \text {; 调用算法 } 3.1 \text {, 计算向量 } P_{i} \text { 的系数向量 } \\
& g=\operatorname{GCD}\left(k_{i, 1}, \ldots, k_{i, n+1}\right) \text {; } \\
& \boldsymbol{k}_{i}=\left(\frac{k_{i, 1}}{g}, \ldots, \frac{k_{i, n+1}}{g}\right) ; \\
& \boldsymbol{d}=\left(D_{1, J}, \ldots, D_{J, J}\right) \text {; 计算矩阵 } \mathrm{D} \text { 的 } J \text { 个代数余子式 } \\
& g=\operatorname{GCD}\left(D_{1, J}, \ldots, D_{J, J}\right) \text {; } \\
& \boldsymbol{d}=\left(\frac{D_{1, J}}{g}, \ldots, \frac{D_{J, J}}{g}\right) ; \\
& \text { for } j \text { from } 1 \text { to } n+1 \\
& K_{j}=\sum_{i=1}^{J} \boldsymbol{d}[i] \cdot \boldsymbol{k}_{i}[j] \\
& g=\operatorname{GCD}\left(K_{1}, \ldots, K_{n+1}\right) \text {; } \\
& \text { for } j \text { from } 1 \text { to } n+1 \\
& K_{j}=\frac{K_{j}}{g} \\
& \operatorname{Res}=\sum_{j=1}^{n+1} K_{j} \cdot f_{j} .
\end{aligned}
$$

上述算法完全去掉了 Dixon 结式的三类多余因子, 即最后的 Res 就是无三类多余因子 的 Dixon 结式.

\section{6 结论}

本文从代数的角度阐明了 Dixon 结式的部分多余因子的产生机理. 显然这种多余因子 的产生机理同样适用于其他结式. 对于 Dixon 结式的退化情形, 即 Dixon 矩阵不为方阵时, 可以按照本文的方法, 先求出每个满足 KSY 条件 ${ }^{[4]}$ 的极大子式, 再求它们的公因式. 
致谢衰心感谢审稿人提出的宝贵意见.

参考文献

1 吴文俊. 初等几何判定问题与机械化证明. 中国科学 A 辑: 数学, 20(6): 507-516 (1977)

2 Buchberger B. Gröbner bases: An algorithmic method in polynomial ideal theory. In: Multidimensional Systems Theory. Dordrecht: Reidel, 1985, 184-232

3 Dixon A L. The eliminant of three quantics in two independent variables. Proc London Math Soc, 6: 49-69, 473-492 (1908)

4 Kapur D, Saxena T, Yang L. Algebraic and geometric reasoning using Dixon resultants, In: Proceedings of the International Symposium on Symbolic and Algebraic Computation. New York: ACM Press, 1994, 99-107

5 符红光, 赵世忠. 构造一般 Dixon 结式矩阵的快速算法. 中国科学 A 辑: 数学, 35(1): 1-14 (2005)

6 Lewis R H, Stiller P F. Solving the recognition problem for six lines using the Dixon resultant. Mathematics and Computers in Simulation, 49(3): 205-219 (1999)

7 Lewis R H. Using Fermat to solve large polynomial and matrix problems. ACM SIGSAM Bulletin, 38(1): $27-28$ (2004)

8 Lewis R H, Coutsias E A. Algorithmic search for flexibility using resultants of polynomial systems. In: Automated Deduction in Geometry. Berlin: Springer, 2006, 186-191

9 张景中, 杨路, 侯晓荣. 代数方程组相关性的一个判准及其在定理机器证明中的应用. 中国科学 A 辑: 数学, 23(10): 1036-1042 (1993)

10 Chionh E W. Rectangular corner cutting and Dixon A-resultants. J Symb Comput, 31(6): 651-669 (2001)

11 Foo M, Chinoh E W. Corner point pasting and Dixon A-resultant quotients. In: Proceedings of the 6th Asian Symposium on Computer Mathematics. Beijing, 2003, 114-127

12 Foo M, Chinoh E W. Corner edge cutting and Dixon A-resultant quotients. J Symb Comput, 37(1): 101-119 (2004)

13 Foo M, Chinoh E W. Dixon A-Resultant quotients for 6-point isosceles triangular corner cutting. In: Geometric Computation. Singapore: World Scientific Publishing, 2004, 374-395

14 Chtcherba A D, Kapur D. Conditions for exact resultants using the Dixon formulation. In: Proceedings of the International Symposium on Symbolic and Algebraic Computation. New York: ACM Press, 2000, $62-70$

15 Chtcherba A D. A new Sylvester-type Resultant Method based on the Dixon-Bezout Formulation. Ph.D. thesis. Albuquerque: The University of New Mexico, 2003

16 Chtcherba A D, Kapur D. A complete analysis of resultants and extraneous factors for unmixed bivariate polynomial systems using the Dixon formulation. In: Kredel H, Seiler W K, eds. Proceedings of the 8th Rhine Workshop on Computer Algebra, 2002, 136-166

17 Chtcherba A D, Kapur D. Exact resultants for corner-cut unmixed multivariate polynomial systems using the Dixon formulation. J Symb Comput, 36(3-4): 289-315 (2003)

18 Chtcherba A D, Kapur D. Resultants for unmixed bivariate polynomial systems produced using the Dixon formulation. J Symb Comput, 38(2): 915-958 (2004)

19 Xiao W, Chionh E W. Formal power series and loose entry formulas for the Dixon matrix. In: Lecture Notes in Computer Science. Berlin: Springer, 2005, 72-82

20 Kapur D, Saxena T. Extraneous factors in the Dixon resultant formulation. In: Proceedings of the 1997 International Symposium on Symbolic and Algebraic Computation. New York: ACM Press, 1997, 141-148

21 Yang L, Hou X R. Gather-and-Sift: A symbolic method for solving polynomial systems. In: Proceeding of First Asian Technology Conference in Mathematics. Singapore: World Scientific Publishing, 1995, 771-780 22 赵世忠. Dixon 结式的理论研究与新算法. 博士学位论文. 北京: 中国科学院研究生院, 2006 